\title{
APPLICATION OF ANALYTICAL SOLUTION FOR EXTENDED SURFACES ON CURVED AND SQUARED RIBS
}

\author{
Tomáš BRESTOVIČ*, Natália JASMINSKÁ*, Marián LÁZÁR \\ *Faculty of Mechanical Engineering, Technical University in Kosice, Department of Power Engineering, \\ Vysokoškolská 4, 04200 Košice, Slovak Republic \\ tomas.brestovic@tuke.sk, natalia.jasminska@tuke.sk, marian.lazar@tuke.sk
}

received 20 October 2014, revised 18 May 2015, accepted 21 May 2015

\begin{abstract}
The presented article discusses how to increase heat transfer through ribbed surfaces and it is oriented to the mathematical representation of temperature fields and the total thermal flow. The complexity of solving for some types of ribs with variable cross-section requires the application of numerical methods, which are applied consequently to the planar rib as well. In this case there was chosen the finite-difference method (FDM). During solution of the cylindrical ribs the FDM method is preferably used directly with regard to the complexity of solving for infinite sums and improper integrals in Bessel functions. In conclusion is assessed the application suitability of the calculation procedure applied to curved ribs. This procedure is usually used to planar ribs. At the same time it is pointed out the possibility of using this method for calculation of the total thermal flow through cylindrical ribs, which have got the squared form.
\end{abstract}

Key words: Temperature Field, Thermal Flow, Numerical Solution, Finite-Difference Method

\section{INTRODUCTION}

Cooling of energy equipments, transport vehicles, as well as electronic components requires intensification of heat transfer from a cooled surface. (Increasing the cooling medium speed OR Increase of cooling medium speed) Increase of the cooling medium speed often does not produce the expected cooling effect. Therefore, the cooling area is increased additionally by means of the newly created ribs. In terms of design the ribs can be either planar or cylindrical. Determination of the total thermal flow for each type of extended surfaces is possible only in the simplified cases usually because a solving of the complex non-linear differential equations of higher order is a task difficult enough. Analysis of the differential equation for a planar rib with a constant rib cross-section, ignoring radiation, enables to obtain the thermal flows and temperature fields using analytical method for various boundary conditions (Maga and Hartanský, 2005).

If there is taken into consideration radiation and dependence of the relevant values on the temperature and on the rib length, it is therefore necessary to use the numerical mathematics. Evidently, the simplest method of numerical solution for ribs seems to be an application of the finite difference method (FDM) (Brestovič and Jasminská, 2013; Pyszko et al., 2010; Purcz, 2001). Application of this method is necessary also for some simple cases. The typical situation is for cylindrical ribs, which temperature fields can be determined by means of Bessel functions. These functions represent solution of improper integrals and infinite sums. That is why it is more suitable to use FDM, which allows to see the changes regard changes of all values in relation to a temperature and a rib length.

Introduction of certain simplified assumption enables to eliminate necessity of solution for two and three dimensional heat conduction. Typical situation is in the case of curved ribbed surfaces or squared ribs. In this situation it is possible to retransform a given task to one-dimensional solution with regard to possible calculation failure. An advantage is a quick solution process (Mlynár and Masaryk, 2012; Ferstl and Masaryk, 2011; Purcz, 2001).

This article offers a complex view of the area of rib design with various types and demonstrates the new solution possibilities for heat transfer using a numerical simulation software.

\section{ANALYTICAL SOLUTION OF HEAT TRANSFER THROUGH EXTENDED SURFACES}

Calculation methodology of thermal flows as well as temperature fields is based on a solution for various types of differential equations obtained from analysis of elementary changes concerning investigated values.

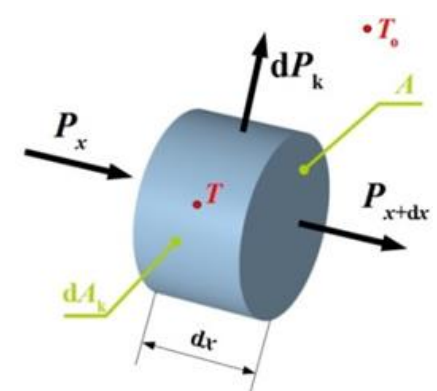

Fig. 1. Thermal flows on the simple rib element

Analytical solution of heat conduction equations is possible only in limited situations, whereas it is applied predominately for stationary one-dimensional heat conduction or for heat conduction with internal sources. More complex geometry volume bodies therefore use software tools based on numerical calcula- 
tions that are far beyond the capabilities of analytical solutions (Stone et al., 2014; Kapjor et al., 2010; Brestovič et al., 2012). A principle of this method consists in a fact that the solution is not required for the whole investigated area, but only for finite number of strategically chosen points (parts of task).

It is necessary to take into consideration several assumptions for definition of temperature fields as well as for thermal flow along the rib length:

1. The heat conduction in the $x$-axis direction is one-dimensional and conduction perpendicular to the $x$-axis is neglected. Isothermal surfaces are perpendicular to the $\mathrm{x}$-axis and their curvature is neglected.

2. The coefficient of heat transfer and the coefficient of thermal conductivity are constant along the whole rib surface.

3. The heat conduction is stationary and the temperature field is constant during time.

According to the thermal flows in the simple rib element, with regard to the law of energy conservation, it is evident that the sum of conductive thermal flow on the output of element and convection from external surface equals to the input of thermal flow to the element:

$P_{x}=P_{x+\mathrm{d} x}+\mathrm{d} P_{\mathrm{k}}$

Fourier law describes a thermal flow due to conduction by relation (Rohsenow et al., 1998; Incropera et al., 2007; Rajzinger, 2012):

$P_{x}=-\lambda \cdot A \cdot \frac{\mathrm{d} T}{\mathrm{~d} x}$

where $A$ is a cross-section area in distance $x\left(\mathrm{~m}^{2}\right), \lambda-$ coefficient of thermal conductivity $\left(\mathrm{W} \cdot \mathrm{m}^{-1} \cdot \mathrm{K}^{-1}\right)$. The conductive thermal flow in distance $\mathrm{x}+\mathrm{dx}$ can be given as follows:

$P_{x+\mathrm{d} x}=P_{x}+\frac{\partial P_{x}}{\partial x} \mathrm{~d} x$

Newton law of heat transfer by convection through the elementary surface $d A_{k}$, which is written in differential form, describes the thermal flow transferred into the surrounding during cooling:

$\mathrm{d} P_{\mathrm{k}}=\alpha \cdot \mathrm{d} A_{\mathrm{k}} \cdot\left(T-T_{\mathrm{o}}\right)(\mathrm{W})$

where $\alpha$ is heat transfer coefficient $\left(\mathrm{W} \cdot \mathrm{m}^{-2} \cdot \mathrm{K}^{-1}\right), d A_{k}-$ elementary surface of rib participated in heat convection $\left(\mathrm{m}^{2}\right)$, $T$ - thermodynamic temperature of the rib element with thickness $d x(K), T_{o}$ - ambient thermodynamic temperature $(K)$.

Joining the relations from (1) to (4) we obtain the relation for energy balance of the thermal flows in the form:

$P_{x}=P_{x}+\frac{\partial\left(-\lambda \cdot A \cdot \frac{\mathrm{d} T}{\mathrm{~d} x}\right)}{\partial x} \mathrm{~d} x+\alpha \cdot \mathrm{d} A_{\mathrm{k}} \cdot\left(T-T_{\mathrm{o}}\right)$

After modification of this equation and using derivation relations we obtain the general differential equation, which describes the rib temperature fields as follows:

$\frac{\mathrm{d}^{2} T}{\mathrm{~d} x^{2}}+\frac{1}{A} \cdot \frac{\mathrm{d} A}{\mathrm{~d} x} \cdot \frac{\mathrm{d} T}{\mathrm{~d} x}-\frac{\alpha}{A \cdot \lambda} \cdot \frac{\mathrm{d} A_{\mathrm{k}}}{\mathrm{d} x} \cdot\left(T-T_{\mathrm{o}}\right)=0$

After calculation of temperature behaviour in dependence on the rib length it is possible to obtain the conductive thermal flow in any distance $x$ according to the relation (2).

Solving of differential equation is possible to perform if there are known geometric, physical and boundary conditions of explicitness (Oravec et al., 2010; Vranay, 2012). The simplest situation for analytic solution of equation is a planar rib with a constant cross-section area.

\subsection{Equation of Energy for Extended Surfaces Considering Radiation}

In case that the rib surface emissivity has not got a zero level (there is considered a grey body), it is necessary to determine the total thermal flow transferred through the rib considering its radiation as well. In the next chapter there is supposed a constant value of emissivity on the whole surface of a sole rib, whereas the ambient effective emissivity equals 1 .

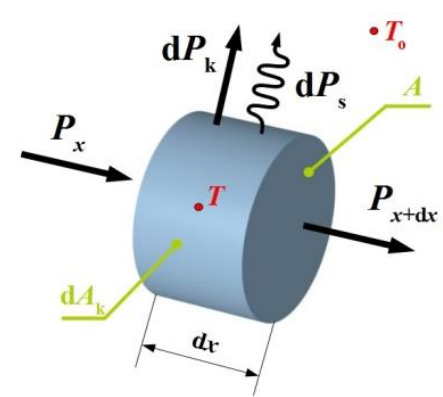

Fig. 2. Description of thermal flows on the simple rib element considering radiation

The equation of the thermal flow, the relation (1), is supplemented with the thermal flow caused by radiation into ambient:

$P_{x}=P_{x+\mathrm{d} x}+\mathrm{d} P_{\mathrm{k}}+\mathrm{d} P_{\mathrm{s}}$

Elementary radiated thermal flow $d P_{S}$ transferred from the rib surface into ambient is determined according to the StefanBoltzmann Law:

$\mathrm{d} P_{\mathrm{s}}=\varepsilon \cdot \sigma \cdot \mathrm{d} A_{\mathrm{k}} \cdot\left(T^{4}-T_{\mathrm{o}}{ }^{4}\right)$

where $\sigma$ is the Stefan-Boltzmann constant $\left(\mathrm{W} \cdot \mathrm{m}^{-2} \cdot \mathrm{K}^{-4}\right), \varepsilon-$ the rib surface emissivity (-), and $d A_{k}$ represents the elementary surface participating on the convention and radiation $\left(\mathrm{m}^{2}\right)$.

Using addition of the relations (7) and (8) and by means of mathematical modification we obtain the final non-linear differential equation of the second order, which describes a onedimensional field of temperature in the rib, considering the radiation.

$\frac{\mathrm{d} A}{\mathrm{~d} x} \cdot \frac{\mathrm{d} T}{\mathrm{~d} x}+A \frac{\mathrm{d}^{2} T}{\mathrm{~d} x^{2}}-\frac{\alpha}{\lambda} \cdot \frac{\mathrm{d} A_{\mathrm{k}}}{\mathrm{d} x} \cdot\left(T-T_{\mathrm{o}}\right)-\frac{\varepsilon \cdot \sigma}{\lambda} \cdot \frac{\mathrm{d} A_{\mathrm{k}}}{\mathrm{d} x}$.
$\left(T^{4}-T_{\mathrm{o}}{ }^{4}\right)=0$

In case of a planar rib with the constant cross-section the equation (9) can be simplified as follows:

$\frac{\mathrm{d}^{2} T}{\mathrm{~d} x^{2}}-\frac{\alpha}{\lambda} \cdot \frac{p}{A} \cdot\left(T-T_{\mathrm{o}}\right)-\frac{\varepsilon \cdot \sigma \cdot p}{\lambda} \cdot\left(T^{4}-T_{\mathrm{o}}{ }^{4}\right)=0$

where $p$ is the perimeter of the rib at a distance $x$ from the base of rib $(m)$.

In view of the problematic solution of these types of differential equations is more convenient to use iterative-numerical calculation using the energy balance of the rib element. To simplify the calculation in the next section is described a calculation procedure using FDM without considering the radiation. 


\section{APPLICATION OF FDM FOR CALCULATION OF THERMAL POWER OUTPUT AND FIELD OF TEMPERATURE FOR PLANAR RIBS}

Determination of the temperature field along the rib height, considering radiation according to the equation (10), is complicated due to a solution of the non-linear differential equation of the second order. At the solution of equation, using the FDM, a set of differential equations is created with the polynomial of the 4th degree. Therefore, it is more suitable to solve the field of temperature using an iterative method with the basic equations describing the conduction and convection. A calculation of the rib temperatures with neglected radiation is realized through equation (6), whereas the first and the second derivations are overwritten by using the Taylor series in the following form (herewith the derivations of the higher order are neglected):

$\frac{\mathrm{d} U}{\mathrm{~d} x}=\frac{U_{i+1}-U_{i}}{\Delta x}$

$\frac{\mathrm{d}^{2} U}{\mathrm{~d} x^{2}}=\frac{U_{i-1}-2 \cdot U_{i}+U_{i+1}}{(\Delta x)^{2}}$

where $U$ is a general variable derivative along the $x$ axis, $U_{i}$ - is a variable in the $i$-th node, $U_{i+1}$ - is a variable in the $(i+1)$ th node, $U_{i-1}-$ is a variable in the $(i-1)$ th node, $\Delta x-$ is a length of the rib partition $(\mathrm{m})$.

In general, it is possible to take into consideration a change of all the relevant quantities along the rib length. If the rib is divided into $\mathrm{n}$ equal elements, it is thus necessary to calculate the $n+$ 1 temperatures that are mutually dependent in the nodal points. With respect to the relations (11) and (12) we obtain a linear equation from the relation (6) in the form:

$\frac{T_{i-1}-2 \cdot T_{i}+T_{i+1}}{(\Delta x)^{2}}+\frac{1}{A_{i}} \cdot \frac{A_{i+1}-A_{i}}{\Delta x} \cdot \frac{T_{i+1}-T_{i}}{\Delta x}$

$-\frac{\alpha_{i} \cdot p_{i}}{A_{i} \cdot \lambda_{i}} \cdot\left(T_{i}-T_{\mathrm{o}}\right)=0$

Using a separation of the searched temperatures, the relation (13) is modified into the form:

$T_{i-1}-\left[2+\frac{A_{i+1}-A_{i}}{A_{i}}+\frac{\alpha_{i} \cdot p_{i}}{A_{i} \cdot \lambda_{i}} \cdot(\Delta x)^{2}\right] \cdot T_{i}+\left[1+\frac{A_{i+1}-A_{i}}{A_{i}}\right]$.

$T_{i+1}=-\frac{\alpha_{i} \cdot p_{i} \cdot(\Delta x)^{2}}{A_{i} \cdot \lambda_{i}} \cdot T_{\mathrm{o}}$

After introducing of a substitution for the coefficients, which are situated in front of the temperatures in the nodal points of the discretised rib, the new form of the relation is:

$T_{i-1}+a_{i} \cdot T_{i}+b_{i} \cdot T_{i+1}=c_{i}$

where $a_{i}, b_{i}$ and $c_{i}$ are constants created by means of the next substitution according to:

$a_{i}=-\left[2+\frac{A_{i+1}-A_{i}}{A_{i}}+\frac{\alpha_{i} \cdot p_{i}}{A_{i} \cdot \lambda_{i}} \cdot(\Delta x)^{2}\right]$

$b_{i}=1+\frac{A_{i+1}-A_{i}}{A_{i}}$

$c_{i}=-\frac{\alpha_{i} \cdot p_{i} \cdot(\Delta x)^{2}}{A_{i} \cdot \lambda_{i}} \cdot T_{\mathrm{o}}$

Equation (15) describes the dependence among the temperature $T_{i}$ in the $i$ - th node and the temperatures $T_{i-1}$ and $T_{i+1}$ in the neighbouring points. The rib is divided into five elements of the same length according to Fig.3; thereby six nodal temperatures are defined. In order to calculate these temperatures it is necessary to assembly the same number of linear equations. Rib is divided into five elements only for purposes of calculation exemplification. Increase the number of partitions would naturally lead to increase in the accuracy of the calculation.

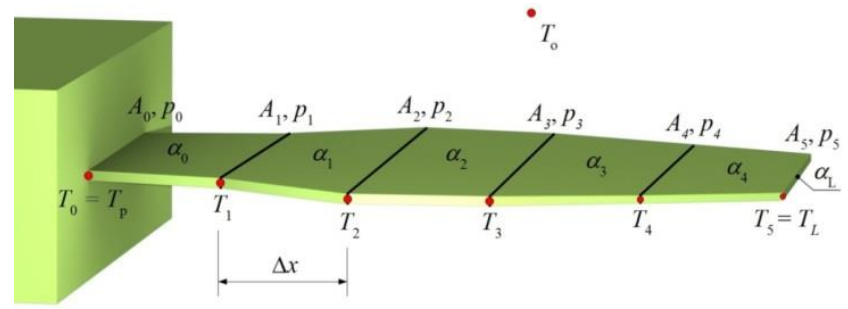

Fig. 3. Illustration of the dipartite rib for FDM

The equation (15) is valid for the nodal points from $i=1$ to 4 , which means the creation of an equation system with 4 ones. The other relations are given by the boundary conditions:

1. The temperature of the rib foot is known $T_{0}=T_{p}=c_{0}$.

2. We consider convection at the rib end, whereas the thermal flow, caused by conduction at the rib end, equals to thermal flow due to convection from the rib end surface into ambient.

$-\lambda \cdot \frac{T_{5}-T_{4}}{\Delta x}=\alpha \cdot\left(T_{5}-T_{\mathrm{o}}\right) \quad\left(\mathrm{W} \cdot \mathrm{m}^{-2}\right)$

Modifying the relation (19) together with following substitution of constants we obtain a relation between the temperature $T_{4}$ and $T_{5}$ in the form:

$\frac{\lambda}{\Delta x} \cdot T_{4}-\left(\frac{\lambda}{\Delta x}+\alpha\right) \cdot T_{5}=-\alpha \cdot T_{\mathrm{o}} \quad\left(\mathrm{W} \cdot \mathrm{m}^{-2}\right)$

$d \cdot T_{4}+e \cdot T_{5}=c_{5}$

$\left(\mathrm{W} \cdot \mathrm{m}^{-2}\right)$

where $d$ and $e$ are the substitution of constants of equation (20).

The system of 6 linear equations can be described in a matrix form:

$|a| \cdot|T|=|c|$

$\left|\begin{array}{llllll}1 & 0 & 0 & 0 & 0 & 0 \\ 1 & a_{1} & b_{1} & 0 & 0 & 0 \\ 0 & 1 & a_{2} & b_{2} & 0 & 0 \\ 0 & 0 & 1 & a_{3} & b_{3} & 0 \\ 0 & 0 & 0 & 1 & a_{4} & b_{4} \\ 0 & 0 & 0 & 0 & d & e\end{array}\right| \cdot\left|\begin{array}{l}T_{0} \\ T_{1} \\ T_{2} \\ T_{3} \\ T_{4} \\ T_{5}\end{array}\right|=\left|\begin{array}{l}c_{0} \\ c_{1} \\ c_{2} \\ c_{3} \\ c_{4} \\ c_{5}\end{array}\right|$

A solution of the equation system roots can be found for example by means of an inverse matrix method:

$|T|=|a|^{-1} \cdot|c|$

A calculation example is realised on the planar rib with the length $50 \mathrm{~mm}$, the thickness $2 \mathrm{~mm}$, the width $100 \mathrm{~mm}$ and the thermal conductivity $\lambda=55 \mathrm{~W} \cdot \mathrm{m}^{-1} \cdot \mathrm{K}^{-1}$.

The coefficient of heat transfer of the rib surface is $\alpha=45 \mathrm{~W} \cdot \mathrm{m}^{-2} \cdot \mathrm{K}^{-1}$, the temperature of surrounding liquid is $T_{0}=20^{\circ} \mathrm{C}$ and the rib partition is $\Delta x=0.01 \mathrm{~m}$.

The solution result of temperature field is a matrix in the form:

$|T|=\left|\begin{array}{l}50.00 \\ 43.65 \\ 39.28 \\ 36.52 \\ 35.13 \\ 35.01\end{array}\right|$ 
In Fig. 4 there is illustrated a comparison of resulting temperature in the nodal points of rib determined by FDM and an analytic solution.

The temperature behaviours along the rib length have got an equal character; however, the low level of rib discretisation by means of large distances $\Delta x$ causes a relevant failure of the calculation using FDM in comparison to an analytic solution.

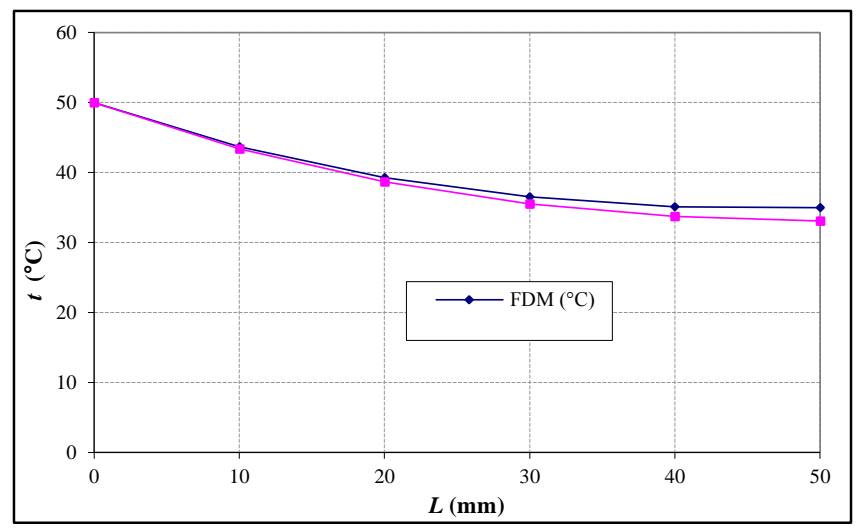

Fig. 4. Behaviour of temperatures along the rib length determined by analytic technique (analytical solution of the equation (6) for planar rib) and by FDM

Calculation deviation considering the actual situation of the rib is $5.84 \%$, whereas if one-dimensional network on the rib surface is more compacted, the calculation deviation decreases.

\subsection{Application of FDM for Calculation of Cylindrical Ribs}

It is more suitable to apply numerical methods for a solution of temperature fields as well as for a solution of the total thermal flow removed through the rib with regard to a complicity and slowness of the analytical solution. Software support for a solution of the cooling power output can be created by a proper implementation of the mathematical methods. Such application is useful predominately in the case of coolers (Čarnogurská et al., 2013, Kapalo, 2005).

The analytical solution is too complicated for a design of quick and easy calculation software. Therefore, it was chosen a calculation method, which is based on FDM application. In this case the cylindrical rib is divided along the height into the $N$ coaxial cylindrical elements. This solution was applied assuming that isothermal surfaces have got a cylindrical shape, although in the case of a real cooler the temperature field is deformed due to gradual air heating along the rib height as well as due to an unequal distribution for the velocity field of cooling air (Fig. 5).

The Fourier law in the differential form describes a conductive heat transfer through a rib and it can be transferred into a difference form according to the equation (27) for a very small change of the radius.

At the numerical method there is used a substitution of the differential equation of the first order by a difference equation (with an application of Taylor series neglecting the second and higher derivations) (Michalec et al., 2010, Nagy et al., 2012, Urban et al., 2012). A requirement for solution by means of FDM is to keep the same division of the radius $r$ during task discretisation, i.e.:

$$
\begin{aligned}
& \frac{\mathrm{d} t}{\mathrm{~d} r}=\frac{T_{i+1}-T_{i}}{r_{i+1}-r_{i}} \quad\left(\mathrm{~K} \cdot \mathrm{m}^{-1}\right) \\
& \text { Then: }
\end{aligned}
$$

$P[i]=-2 \cdot \pi \cdot r_{i} \cdot \delta \cdot \lambda \cdot \frac{T_{i+1}-T_{i}}{r_{i+1}-r_{i}}$

where: $r$ is the internal radius of the $i$-th element $(\mathrm{m}), r_{i+1}$ is the external radius of the $i$-th element $(\mathrm{m}), T_{i}$ is the temperature of the i-th element $(K)$, and $T_{i+1}$ is the temperature of the $(i+$ 1)-th element $(K)$.

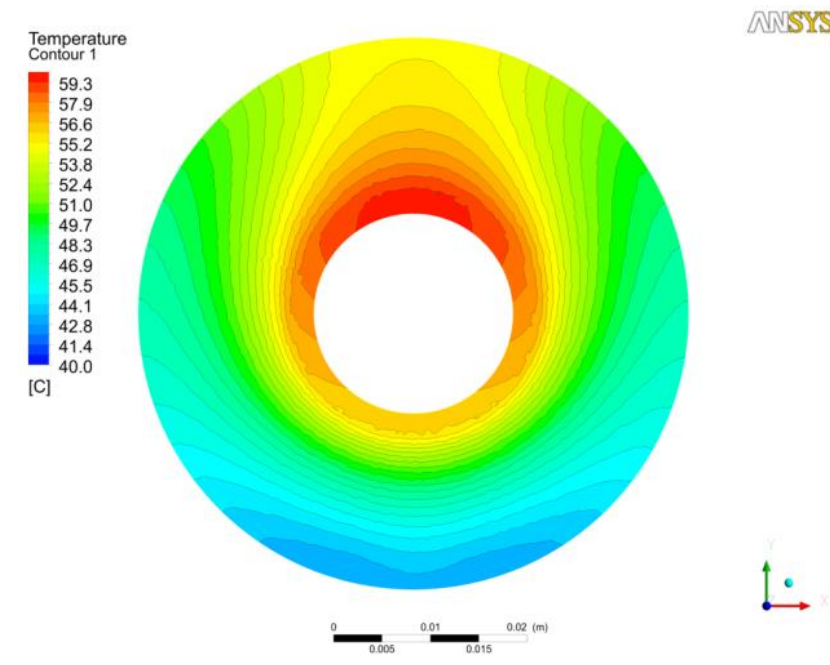

Fig. 5. Temperature field on the rib during flowing of the cooling liquid in $y$-axis direction

The Newton law describes a heat transfer from a rib surface into the air:

$P_{\mathrm{k}}[i]=\alpha \cdot\left(T_{i}-T_{\mathrm{o}}\right) \cdot \pi \cdot\left(r_{i+1}^{2}-r_{i}^{2}\right)$

where $T_{\mathrm{O}}$ is the cooling air temperature $(K)$.

The relation (29) describes the conductive thermal flow entering the next $[i+1]$-th element, whereas the calculation has to fulfil the basic boundary conditions:

$P[i+1]=P[i]-2 \cdot P_{\mathrm{k}}[i]$

The 1st boundary condition: $r[0]=r_{1} \Rightarrow t[0]=t_{\mathrm{p}}$. The 2nd boundary condition: $P_{\mathrm{k}}[N]=2 \cdot \alpha \cdot \pi \cdot r_{2} \cdot \delta \cdot\left(T_{N}-T_{\text {air }}\right)$ (convection at the rib end), where $T N$ is a temperature of the boundary $N$-th element $(K), T_{\text {air }}$ - temperature of the surrounding fluid medium $(K)$.

It is necessary to estimate the thermal flow as accurately as possible for the first iteration at the rib foot in order to accelerate the iterative calculation.

This requirement can be obtained by a calculation of the planar rib with the constant cross-section, whereas a rib crosssection and a rib circumference is considered at the middle radius of the cylindrical rib:

${ }_{1} P[0]=\sqrt{\alpha \cdot \lambda \cdot p_{\text {avg }} \cdot A_{\text {avg }}} \cdot\left(T_{\mathrm{o}}-T_{\mathrm{vz}}\right) \cdot$
$\tanh \left[\sqrt{\frac{\alpha}{\lambda} \cdot \frac{p_{\text {avg }}}{A_{\text {avg }}}} \cdot\left(r_{2}-r_{1}\right)\right] \quad$ (W)

where: $p_{\text {avg }}$ is the perimeter of the planar rib corresponding to the mean radius of the cylindrical rib (m), $A_{\text {avg }}$ is a cross-sectional area on the mean radius of the rib $\left(\mathrm{m}^{2}\right)$. 


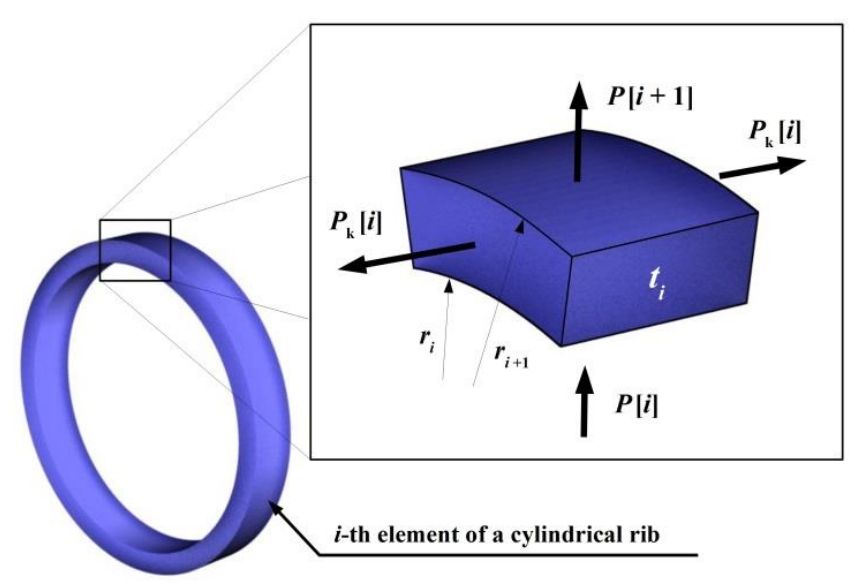

Fig. 6. Thermal flows in the element of a cylindrical rib

The calculation is performed iteratively; at the end of each individual iteration it is necessary to change an initial conductive thermal flow at the rib foot $P_{\mathrm{k}}[0]$ so that the boundary condition No. 2 will be reached. For this purpose there was suggested an iterative condition. During application of this condition it was investigated that its calculation is still converging to the required real value:

${ }_{j+1} P[0]={ }_{j} P[0]-\frac{{ }_{j} P[N]}{10}$

where: ${ }_{j} P[0]$ - is the thermal conductive flow at the rib input for the $j$-th iteration $(\mathrm{W}),{ }_{j+1} P[0]-$ is the thermal conductive flow at the rib input for the $(j+1)$-th iteration, and ${ }_{j} P[N]-$ is the thermal flow at the external rib radius for $j$-th iteration $(\mathrm{W})$.

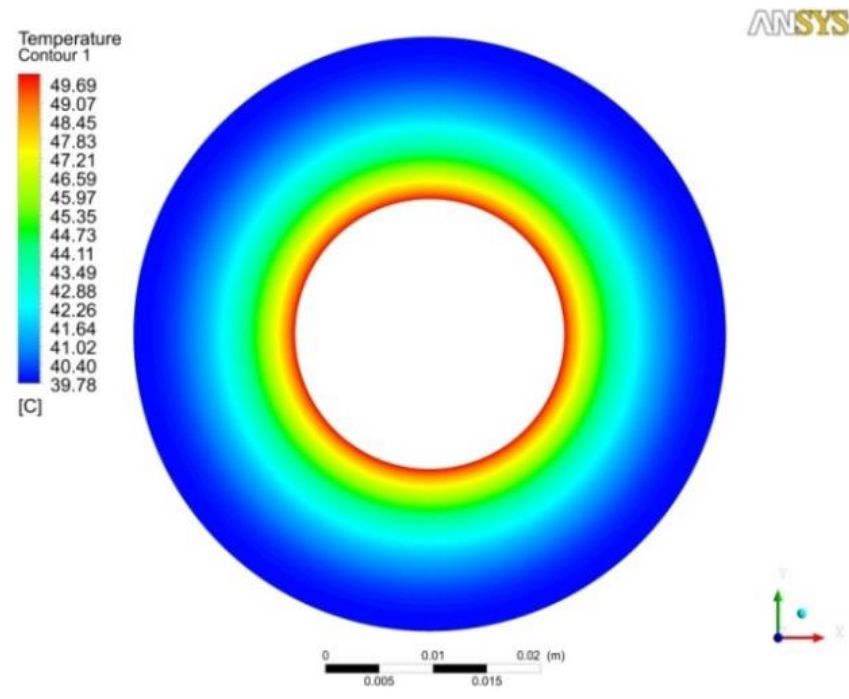

Fig. 7. Temperature field on a cylindrical rib of the natural gas cooler CH_R (ANSYS CFX)

The results of calculation obtained by means of the abovementioned method were compared using the simulation tool ANSYS CFX (Fig. 8) for the same boundary conditions:

1. The rib foot temperature: $50^{\circ} \mathrm{C}$.

2. The coefficient of heat transfer on the rib: $45 \mathrm{~W} \cdot \mathrm{m}^{-2} \cdot \mathrm{K}^{-1}$ (at ambient air temperature $20^{\circ} \mathrm{C}$ ).

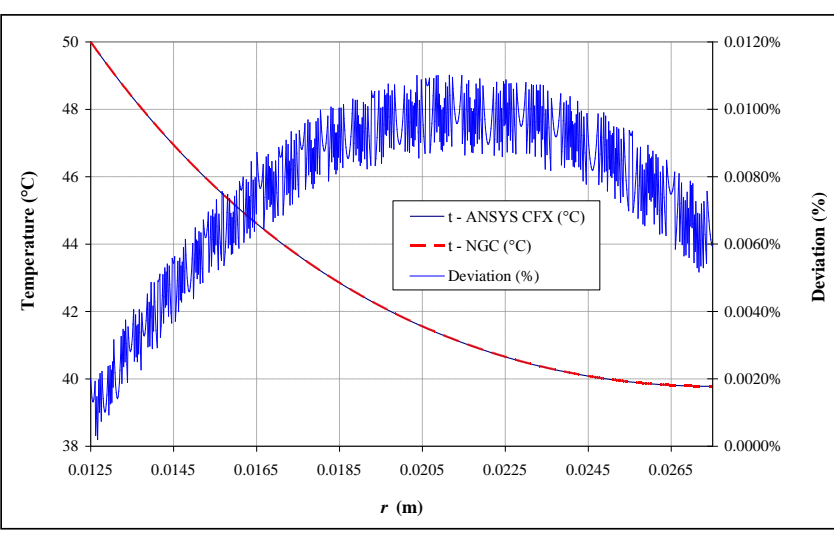

Fig. 8. A comparison of the temperature behaviours along the rib height obtained with various numerical tools

The deviations of the temperature behaviours along the rib height are up to the maximum value $0.015 \%$ (Fig. 8). These temperature behaviours can be obtained either by the numerical calculation, which is performed by the newly developed software NGC (Natural Gas Cooler) or by the commerce software ANSYS CFX.

\section{APPLICATION OF ANALYTICAL SOLUTION CONCERNING PLANAR RIBS FOR CURVED RIBS}

The analytical and numerical calculation of the planar and cylindrical ribs was realised providing one-dimensional stationary heat conducting. In practical applications there is heat conducted through extended surfaces with a constant crosssection, whereas the centre of gravity for a surface, which conductive thermal flow is passing through, often creates a general curve. Therefore, it is not possible to consider the planar ribs. However, there is an advantageous possibility to apply mathematical functionalities deduced for the planar ribs. A typical example of a curved body is a handle of a fire stove specified for dendromass combustion, (Fig. 9). Although this handle does not fulfil a rib function, the thermal flow, which is passing through it, can be solved by means of assumptions valid for the planar ribs.

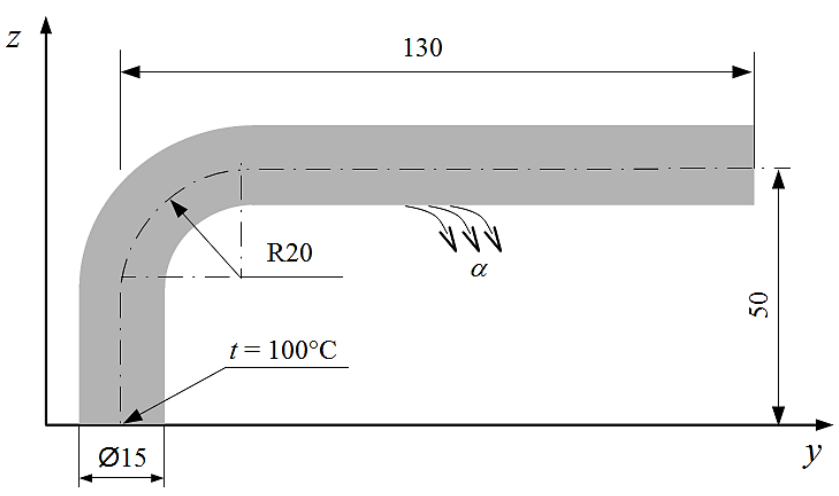

Fig. 9. The geometrical and thermal boundary conditions of the handle

A suitability of the assumption for calculation of the curved ribs with the constant cross-section was verified by a comparison. This comparison was realized between the analytical calculation of the rib end temperature considering the total thermal flow and the second calculation, which was fulfilled using finite volume method 


\section{(FVM) in ANSYS CFX.}

The rib foot temperature is $100^{\circ} \mathrm{C}$, the ambient air temperature is $25^{\circ} \mathrm{C}$ and the end of the handle is adiabatically insulated.

A gravity point axis of the cross-section surface is situated horizontally. The heat transfer coefficient $\alpha=8.844 \mathrm{~W} \cdot \mathrm{m}^{-2}$. $\mathrm{K}^{-1}$ is determined by means of the criterion equations that are valid for heat transfer during free convection and by the known geometrical and physical characteristics of the handle and ambient air.

The HTC (Heat Transfer Coefficient) software was developed in order to quicken a calculation of the heat transfer coefficient. There is considered the rib thermal conductivity value of $60.5 \mathrm{~W}$. $\mathrm{m}^{-1} \cdot \mathrm{K}^{-1}$ and the surface emissivity value $\varepsilon \approx 0$ (this assumption is correct for a chromium-plated surface).

After the solution and modification of the equation (6) we obtain a calculating relation of the handle end temperature in the form:

$T_{L}=T_{\mathrm{o}}+\left(T_{\mathrm{p}}-T_{o}\right) \cdot \frac{1}{\cosh (m \cdot L)}$

where $m$ is the substitution of constants resulting from the analytical solution of the differential equation for the extended area $\sqrt{\frac{\alpha}{\lambda} \cdot \frac{p}{A}}\left(\mathrm{~m}^{-1}\right), L-$ length of the rib $(m)$.

The analytical calculation, performed according to the relation (32), determines the temperature value $T_{L}=71.026^{\circ} \mathrm{C}$. After a solution of the equation (6) with applying the Fourier's law, there is the value level of thermal flow, which is removed through the handle, $P_{r}=3.953 \mathrm{~W}$. As well using ANSYS CFX a calculation was performed in order to compare the obtained results.

The calculated rib end temperature was $T_{L-A N S Y S}=$ $71.148{ }^{\circ} \mathrm{C}$ and the total thermal flow was $P_{r-A N S Y S}=$ $3.956 \mathrm{~W}$. The percentage deviation between the analytical calculation and FVM is $\Delta_{T}=0.17 \%$ for the temperature values and the percentage deviation for the thermal flow is $\Delta_{P}=0.076 \%$. It is evident, with regard to the above-mentioned deviations, that the applied assumptions are correct for an analytical calculation of the planar ribs. In Fig. 10 there are illustrated the isothermal surfaces of the handle cross-section calculated in ANSYS CFX

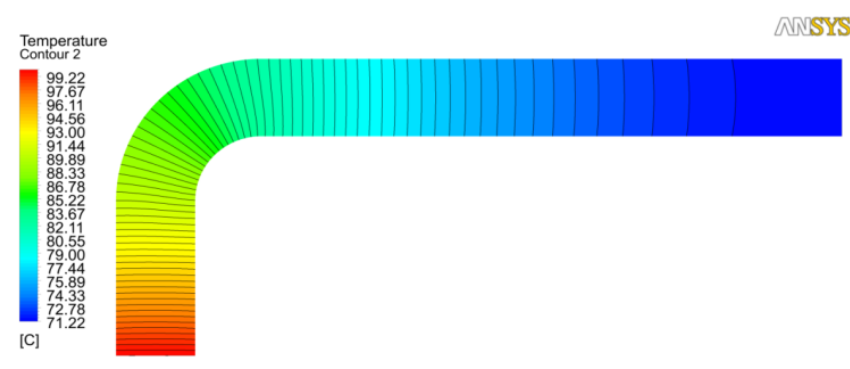

Fig. 10. Thermal field in the plane passing through a middle of handgrip handle

The calculation demonstrates a fact that the isothermal surfaces are not curved in the location of the bar deflection. This fact is favourable for an assumption of an analytical calculation of heat transfer because an increase of convective thermal flow on the external radius is compensated by a reduction of the thermal flow on the internal surface of the handle curvature.

An evaluation of a curvature impact on accuracy of the analyt- ical calculation for a thermal flow passing through a planar rib was realised also for another type of geometry by means of the simulation tool ANSYS CFX. Another geometry consisted from one thread of a helix with such curvature radius, which equals to the pitch $R=h=50 \mathrm{~mm}$. After a planar rollout of the helix a right triangle is created with the side value $2 \cdot \pi \cdot R$ and the pitch $h$.

The hypotenuse of this triangle represents a length $L$ of a spatial curve, which passes through the middle of a helix profile.

$L=\sqrt{(2 \cdot \pi \cdot R)^{2}+h^{2}}$

The equivalent thermal flow of the planar rib, which is made from aluminium, will be solved according to the equation (6) for this geometry with the length $L$ and for the adiabatic rib end. The value of rib heat conductivity is considered $\lambda=237 \mathrm{~W} \cdot \mathrm{m}^{-1}$. $\mathrm{K}^{-1}$ and the heat transfer coefficient value is $\alpha=50 \mathrm{~W}$. $\mathrm{m}^{-2} \cdot \mathrm{K}^{-1}$ at the ambient temperature $20^{\circ} \mathrm{C}$. The defined rib foot boundary condition of the first type is $t_{p}=50^{\circ} \mathrm{C}$.

The cross-sectional area of a profile is a cyclic one with the diameter $d$. A change of this diameter causes a change of the ratio $R / d$ as well. The calculation was performed for the ratio values $R / d=20$ (Fig. 11) $R / d=10$ (Fig. 12), $R / d=5$ (Fig. 13), $R / d=3$ (Fig. 14), $R / d=1.5$ (Fig. 15) and $R / d=1$ (Fig. 16).

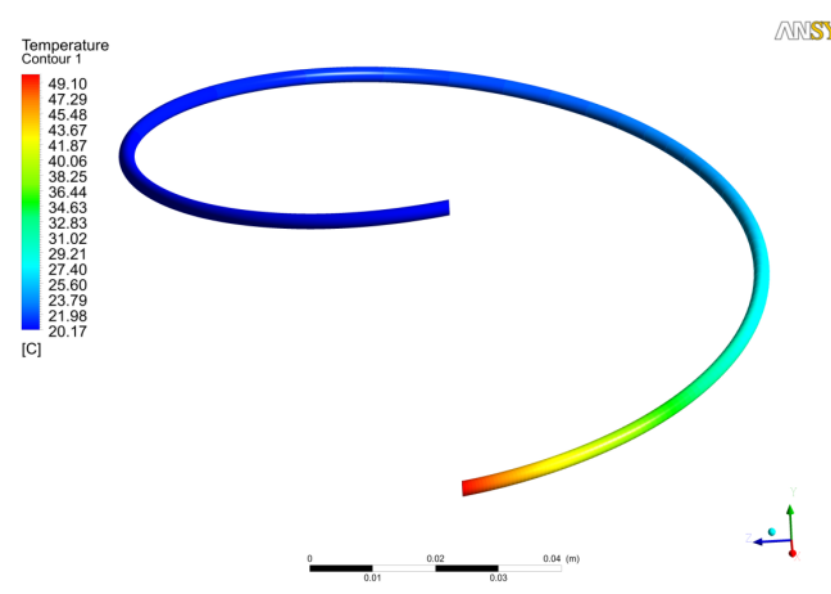

Fig. 11. Surface temperature field of curved bar with the ratio $R / d=20$

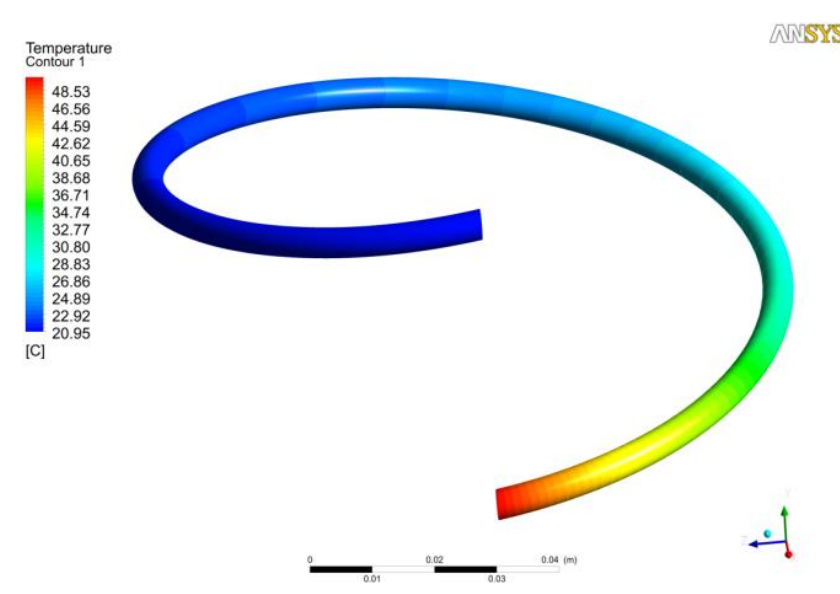

Fig. 12. Surface temperature field of curved bar with the ratio $R / d=10$ 


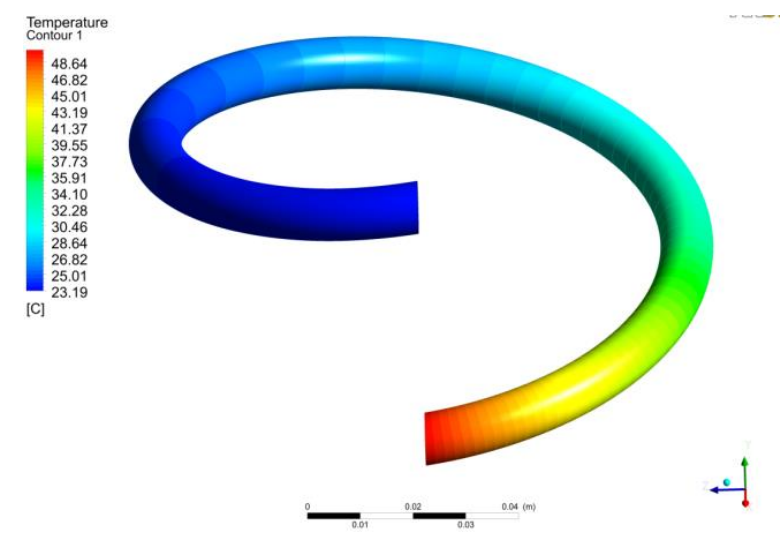

Fig. 13. Surface temperature field of curved bar with the ratio $R / d=5$

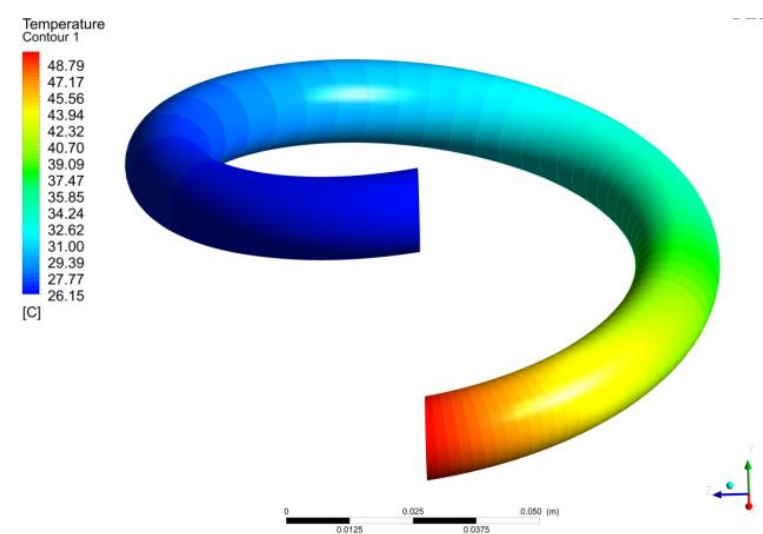

Fig. 14. Surface temperature field of curved bar with the ratio $R / d=3$

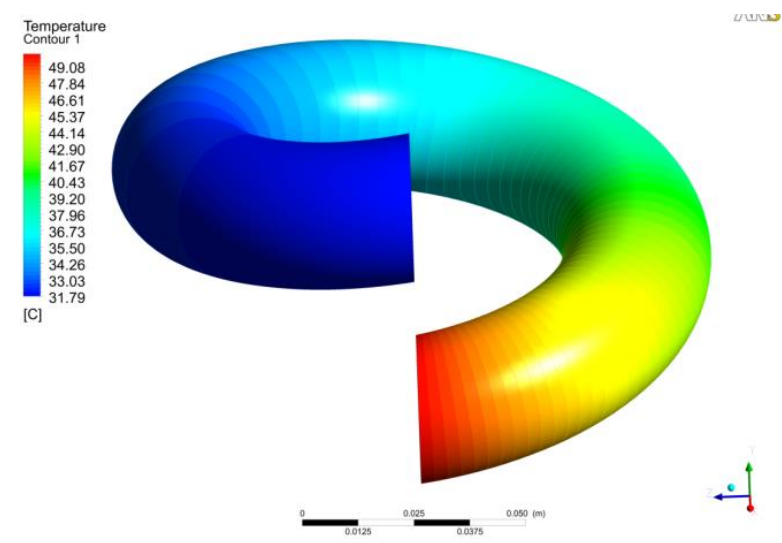

Fig. 15. Surface temperature field of curved bar with the ratio $R / d=1.5$

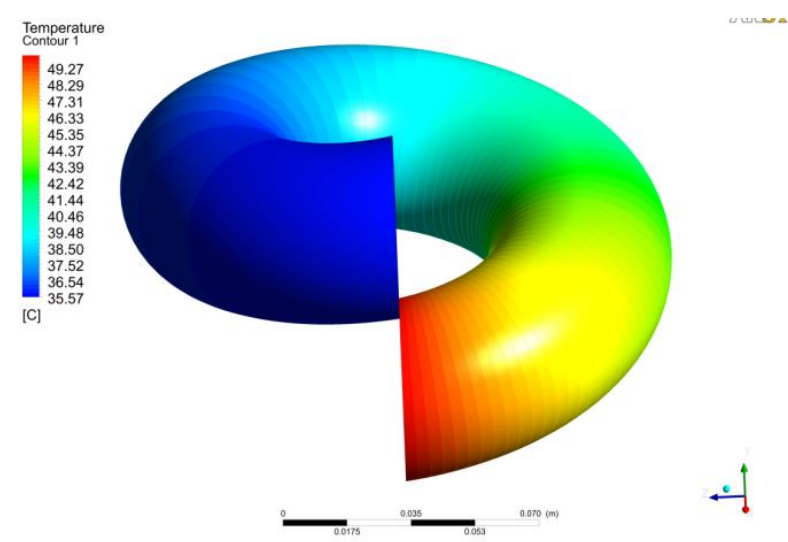

Fig. 16. Surface temperature field of curved bar with the ratio $R / d=1$
Tab.1 presents a comparison of the total thermal flow values $P_{r}$ obtained by means of the analytical calculation and the values $P_{r-A N S Y S}$, which are resulting from the calculation using the software ANSYS CFX. In this table there are also recorded the calculating deviations corresponding to the both methods.

Tab. 1. Comparison between the analytical calculation and the calculation using FVM

\begin{tabular}{|c|c|c|c|}
\hline $\begin{array}{c}\boldsymbol{R} / \boldsymbol{d} \\
(-)\end{array}$ & $\begin{array}{c}\boldsymbol{P}_{\boldsymbol{r}} \\
(\mathbf{W})\end{array}$ & $\begin{array}{c}\boldsymbol{P}_{\boldsymbol{r}-\text { ANSYS }} \\
(\mathbf{W})\end{array}$ & $\begin{array}{c}\text { Calculating Deviation } \\
(\mathbf{\%})\end{array}$ \\
\hline $\mathbf{2 0}$ & 0.641 & 0.634 & 1.147 \\
\hline $\mathbf{1 0}$ & 1.813 & 1.790 & 1.269 \\
\hline $\mathbf{5}$ & 5.100 & 5.057 & 0.847 \\
\hline $\mathbf{3}$ & 10.801 & 10.707 & 0.865 \\
\hline $\mathbf{1 . 5}$ & 28.776 & 28.797 & -0.070 \\
\hline $\mathbf{1}$ & 49.523 & 50.025 & -1.012 \\
\hline
\end{tabular}

A deviation of the both calculation methods does not overreach the value of $1.269 \%$. So it can be concluded that the above-mentioned method, which substitutes a curved rib calculation by a calculation of planar rib, is a suitable procedure. An inaccuracy of the calculation can be also caused due to the object discretisation by means of FVM.

\section{CALCULATION OF POWER OUTPUT FOR FLAT SQUARED RIBS}

A heat conduction of flat squared ribs is a two-dimensional process and, as a result, the isothermal surfaces are "deformed" predominately on the external rib side. (Fig.18). Comparison of the temperature fields, for a cylindrical rib and a squared rib, demonstrates a fact that the temperature field is similar in such area where the temperature difference between a surface and surrounding is maximal (on an internal diameter).

The squared rib temperature field is deforming with an increasing distance of a rib element from the central axis. In these areas the surface temperature is lower. An influence of thermal isotherm deformations (curvature) on the total thermal flow, which is determined by an analytical solution of cylindrical ribs, is neglecting in the case of a procedure applied for the squared ribs.

This consideration is verified by a numerical calculation of squared ribs using ANSYS CFX and by a calculation of cylindrical ribs using the own software (FDM). The numerical calculation of cylindrical ribs was also performed in ANSYS CFX in order to compare the temperature fields.

A substitution of squared ribs by cylindrical ribs is possible only on a condition that the frontal surface is the same.

As well as the internal diameter $d$ is identical in both cases. The relation between the square side length and an external diameter of cylindrical rib is given:

$a=\sqrt{\pi} \cdot \frac{D}{2} \quad(\mathrm{~m})$

where $a$ is a square side length $(\mathrm{m}), d$ is an internal diameter of rib $(\mathrm{m})$, and $D$ is an external diameter of the cylindrical rib (m).

Various ratio values of diameters $D / d$ were investigated in order to evaluate a suitability of the above-mentioned assumptions. The ratios $D / d$ are corresponding with an adequate ratio value $a / d$ according to the relation (34). A simulation calculation 
of a temperature field for a cylindrical rib with the ratio $D / d=2$ is illustrated in the Fig. 17.
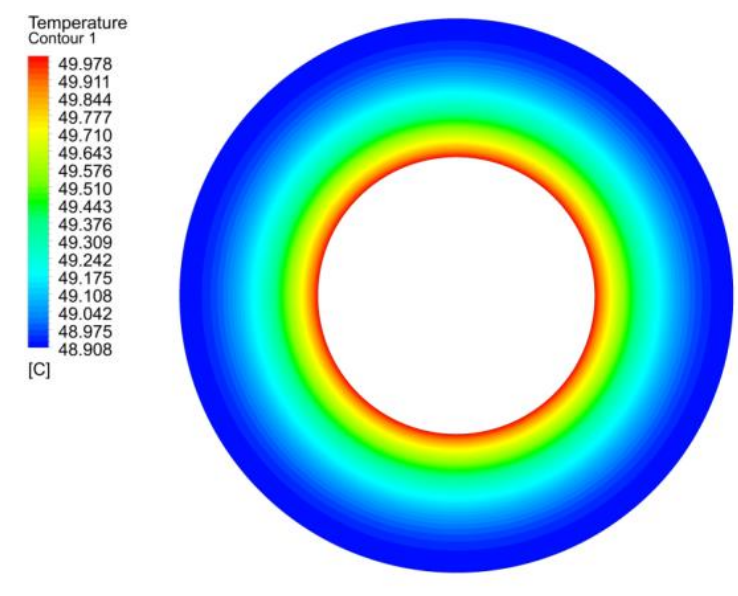

$$
{ }_{0.0025} \stackrel{0.005}{0.0075}^{0.01(m)}
$$

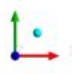

Fig. 17. Temperature field of a cylindrical rib with the ratio of diameters $D / d=2$
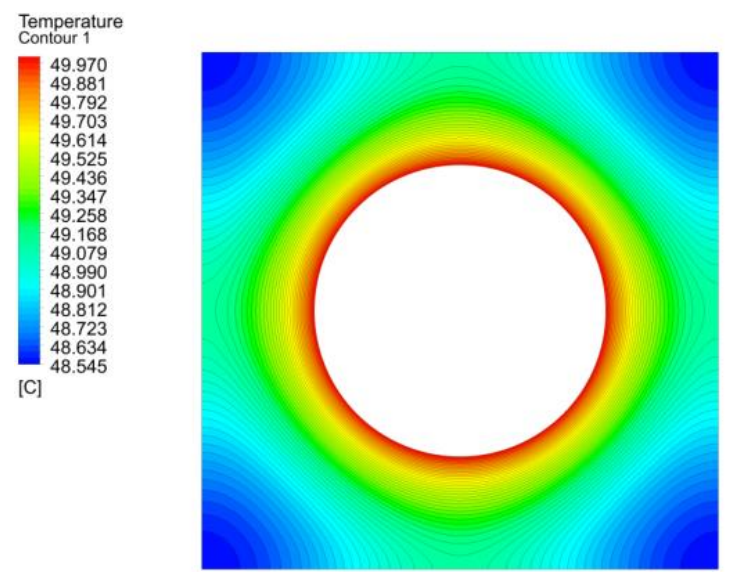

$$
0.0025 \stackrel{0.005}{0.0075}^{0.01(m)}
$$

Fig. 18. Temperature field of a squared rib corresponding to the ratio $D / d=2$
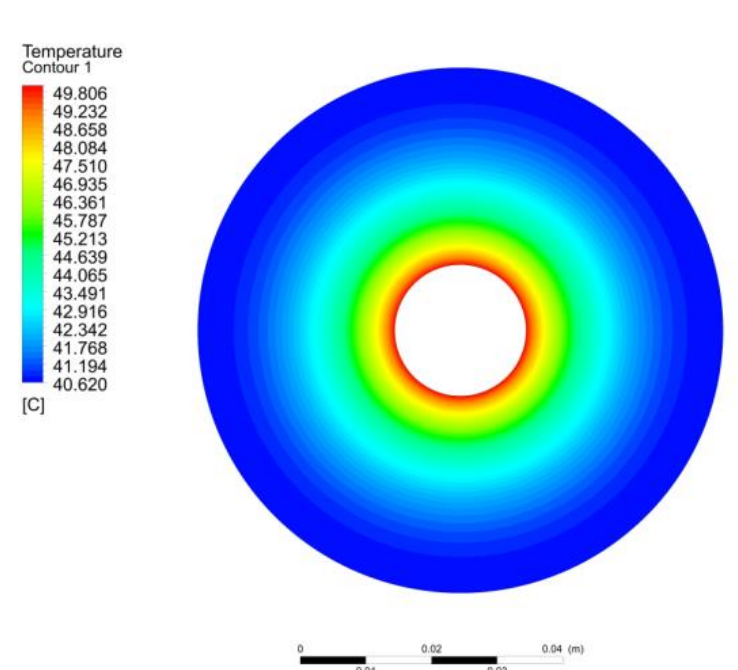

Fig. 19. Temperature field of a cylindrical rib with the ratio of diameters $D / d=4$

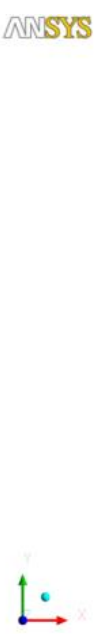

Temperature
Contour 1
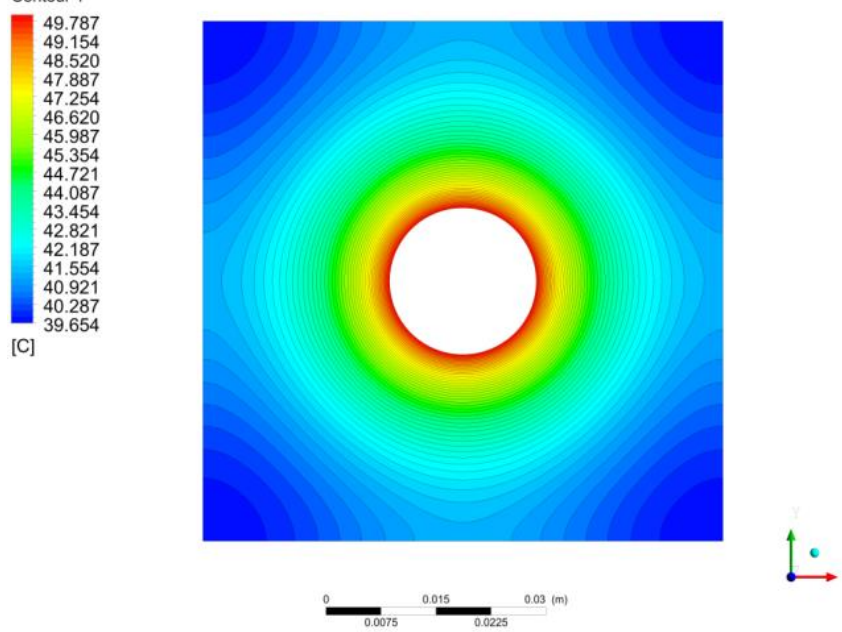

Fig. 20. Temperature field of a squared rib corresponding to the ratio $D / d=4$
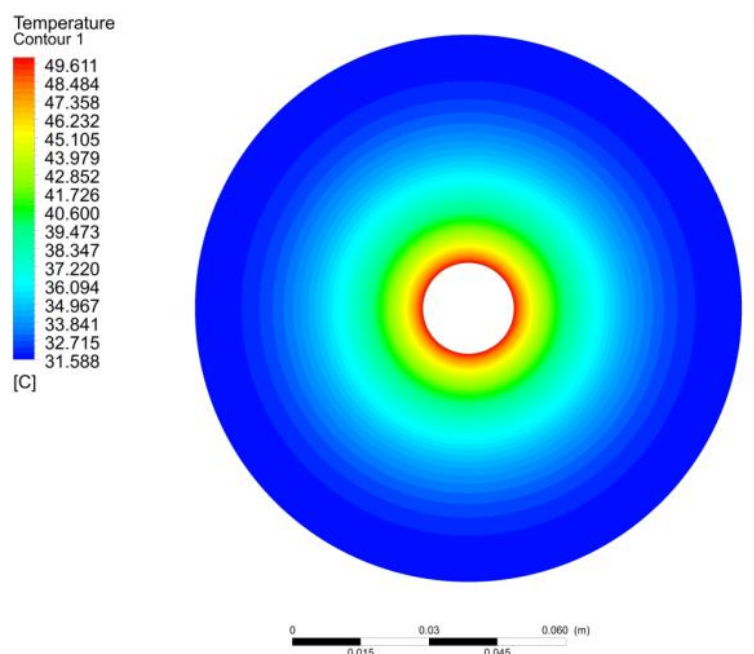

Fig. 21. Temperature field of a cylindrical rib with the ratio of diameters $D / d=6$
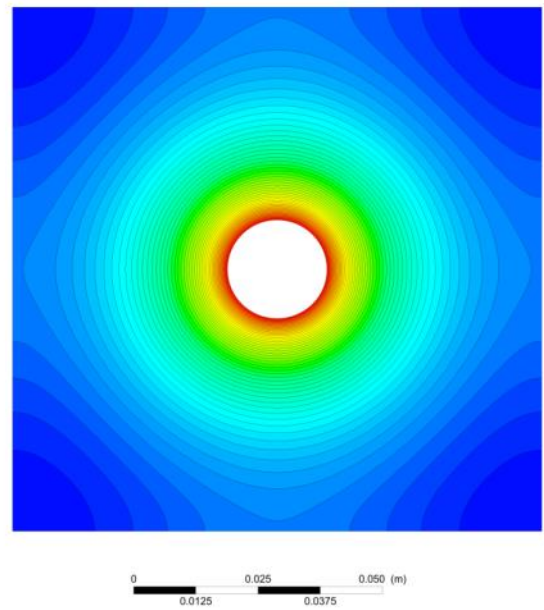
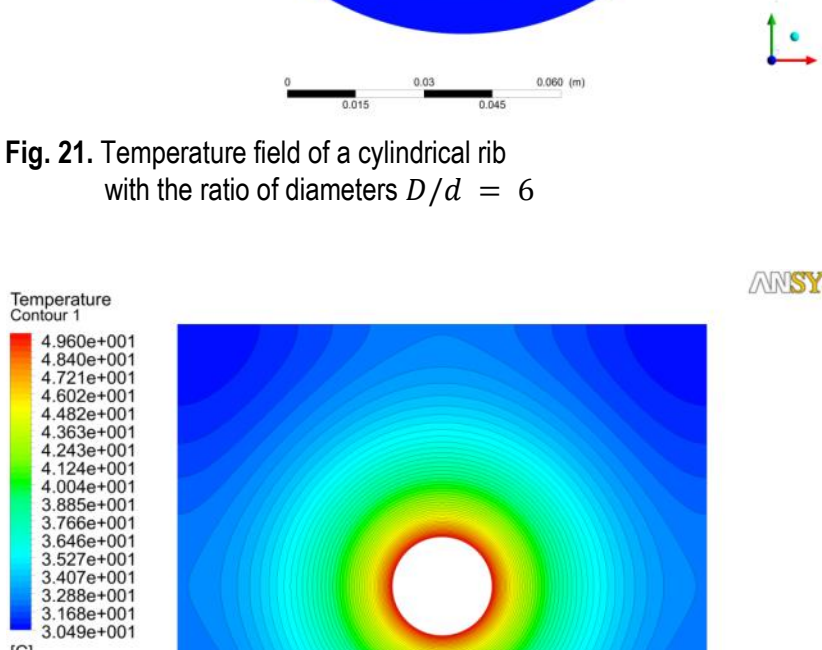

Fig. 22. Temperature field of a squared rib corresponding to the ratio $D / d=6$ 
There is the corresponding ratio $a / d=1.772$ for the squared rib (Fig. 18). The next figures from the Fig. 19 to the Fig. 22 illustrate the temperature contours for the ratios $D / d=4$ and $D / d=6$.

The value of rib heat conductivity is considered $\lambda=237$ $\mathrm{W} \cdot \mathrm{m}^{-1} \cdot \mathrm{K}^{-1}$ and the heat transfer coefficient value is $\alpha=35 \mathrm{~W} \cdot \mathrm{m}^{-2} \cdot \mathrm{K}^{-1}$ at the ambient temperature $20^{\circ} \mathrm{C}$. The defined rib foot boundary condition of the first type is $t_{p}=$ $50{ }^{\circ} \mathrm{C}$. In the Tab. 2 there is presented comparison of the total thermal flow removed through a cooling cylindrical rib and a squared rib. The cylindrical rib was calculated using the own developed software, which applies the finite difference method FDM.

Tab. 2. The comparison of an analytical calculation with a ANSYS CFX calculation

\begin{tabular}{|c|c|c|c|}
\hline $\begin{array}{c}\boldsymbol{D} / \boldsymbol{d} \\
(-)\end{array}$ & $\begin{array}{c}\text { Squared rib } \\
\boldsymbol{P}_{\boldsymbol{r}}(\mathbf{W})\end{array}$ & $\begin{array}{c}\text { Cylindrical rib } \\
\boldsymbol{P}_{\boldsymbol{r}}(\mathbf{W})\end{array}$ & $\begin{array}{c}\text { Calculating deviation } \\
(\mathbf{\%})\end{array}$ \\
\hline $\mathbf{2}$ & 1.922 & 1.926 & -0.179 \\
\hline $\mathbf{4}$ & 7.345 & 7.373 & -0.388 \\
\hline $\mathbf{6}$ & 10.972 & 11.017 & -0.411 \\
\hline
\end{tabular}

An application suitability of an analytical calculation for thermal flow, which is removed through a cylindrical rib, for a calculation of a flat squared rib was verified on the basis of relatively small calculating deviations of power output values in comparison to the simulations performed in ANSYS CFX.

A gradual rising of deviation absolute values in a calculation process is caused due to a substitution of a cylindrical rib by a squared rib. Both ribs have got the same frontal surface area. This fact causes a rising of distance between the rib axis and the square edge in comparison to the width of a cylindrical rib. However, the rising distance also causes a decrease of surface temperature and in this way the total thermal flow is reduced, which is transferred by convection into environment.

\section{CONCLUSIONS}

This article is focused on the methodology, which is specified for a design of planar and cylindrical ribs using analytical and a numerical method. Application of an analytical calculation is possible predominately in the case of simpler geometrical shapes and also for simpler accepted boundary conditions.

Numerical methods are suitable especially for more complicated geometrical shapes and for a creation of a functional dependence among the relevant values and temperatures. In such situations there are applied more difficult differential equations that are used for a solution of heat transfer through ribs.

The mostly used method for a solution of the abovementioned problems is the method of finite differences. This method is a base for the newly developed software tools specified for a calculation of the ribbed surfaces.

In this article there were also described procedures that enable to re-transform the multi-dimensional tasks of the heat transfer through ribs into the one-dimensional tasks.

The main objective for investigation of a transformation possibility of the multi-dimensional tasks into the one-dimensional task is to find simpler tools. Those tools would provide results that are comparable with the results obtained by using various commercial tools, which may not be available for every user.

\section{REFERENCES}

1. Brestovič T., Jasminská N. (2013), Software support development for numerical solution of Ansys CFX, Acta Mechanica et Automatica. Vol. 7, no. 4, 215-221.

2. Brestovič T., Kubík M., Jasminská N. (2012), Use of numerical methods for determining the coefficients of pressure losses (in Slovak), Plynár-Vodár-Kúrenár + Klimatizácia, Vol. 10, č. 2, 46-47.

3. Čarnogurská M., Příhoda M., Kubík M., Gállik R., Hršák D. (2013), Methodology of the Sediment Thickness Calculation on the Heat Exchange Area of a Coolers Natural Gas, International Journal of Mechanic Systems Engieneering, Vol. 3, no. 1, 14-19.

4. Ferstl K., Masaryk M. (2011), Heat transfer (in Slovak), STU Bratislava.

5. Incropera F. P. et al. (2007), Fundamentals of Heat and Mass Transfer, Publisher John Wiley \& Sons.

6. Kapalo P. (2005), The effect of the flow rate of hot water to the heat flow through the pipe wall (in Slovak), TZB, Vol. 13, No. 3, 22-24.

7. Kapjor A., Jandačka J., Malcho M., Papučík Š. (2010), Intensification of Heat Transport from the Floor Convector at Given Geometry and the Way of Use, Meeting's of the department of fluid mechanics and thermodynamics - International Conference, 101 - 104.

8. Maga D., Hart'anský R. (2005), Numerical solutions (in Slovak), Brno.

9. Michalec Z., Taraba B., Bojko M., Kozubková M. (2010), CFD modelling of the low-temperature oxidation of coal, Archivum Combustions, Vol. 30, №. 3, 133-144.

10. Mlynár P., Masaryk M. (2012), Optimalization of absortioption cooling unit, Gépeszet 2012, $8^{\text {th }}$ International conference of Mechanical Engineering, BME Budapest, 361-365.

11. Nagy R., Košičanová, D. (2012), Indoor environment air quality ventilation rates - numerical CFD simulations calculations and measuring apparatus applications, Czasopismo Techniczne, 109 (3), 2012, 281-295.

12. Oravec M., Števo S., Sekaj I. (2010),Comparison of Using Simple Genetic Algorithm and Parallel Genetic Algorithm in Heat Transfer Model Optimization, Journal of Cybernetics and Informatics, Vol. 9 , 13-18.

13. Purcz P. (2001), Parallel algorithm for spatially one- and twodimensional initial-boundary-value problem for a parabolic eauation, Kybernetika, Vol. 37, No. 2, 171-181.

14. Pyszko R., Příhoda M., Velička M. (2010), Method for determining the thermal boundary condition in the $\mathrm{CC}$ mould for numeric models, Proceedings of 19. conference METAL 2010, Ostrava.

15. Rajzinger J. (2012), Calculation of maximum water content in various natural gases by using modified Peng-Robinson equation of state, Communications, Vol. 14, No. 4A, 29-35.

16. Rohsenow W. M., Hartnett J. P., Cho Y. I. (1998), Handbook of Heat Transfer, McGraw-Hill, 250-257

17. Stone C., Simiček J., Vranay F. (2014), Assessing the Thermal Stability of Rammed Earth Using Finite Element Methods, Progressive Multifunctional Building Materials, Constructions and Technological Methods - One Step Closer to Green Visegrad in V4 Countries - Kosice, 107-118.

18. Urban F., Kučák L'., Bereznai J., Pulmann M., Tihányi J. (2012), Influence of the mixing grid position on the coolant flow at the outflow part of the nuclear reactor fuel assembly physical model and validation of CFD model, Communications, Vol. 14, No. 4A (2012), 42-46.

19. Vranay F. (2012), Hydraulic connection with the use of existing heating distribution for cooling) (in Slovak), Vykurovanie, 499-503.

This paper was written with the financial support of the granting agency VEGA of the Ministry of Education of the Slovak Republic within the project solution No. 1/0686/13 and of the granting agency KEGA of the Ministry of Education of the Slovak Republic within the project solution No.041TUKE-4/2013. 